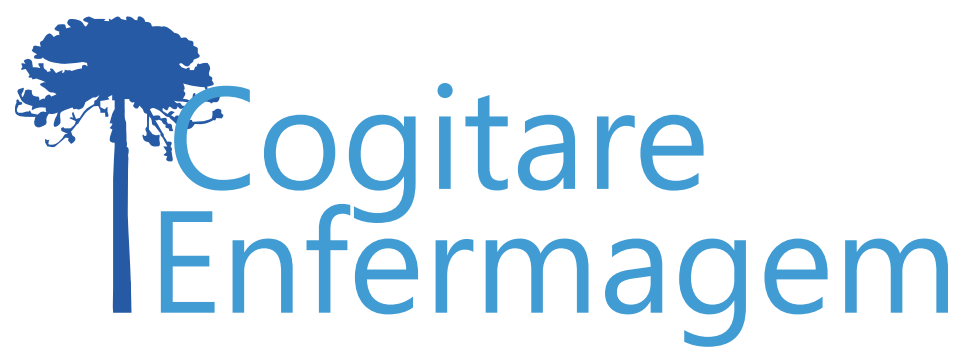

\title{
ANÁLISIS DEL MODELO DE GESTIÓN DE CASOS PARA PERSONAS EN SITUACIÓN DE PLURIPATOLOGÍA Y CUIDADORES FAMILIARES
}

\section{ANALYSIS OF THE CASE MANAGEMENT MODEL FOR PEOPLE WITH MULTIMORBIDITY AND FAMILY CAREGIVERS}

\author{
Ruth Alexandra Castiblanco Montañez ${ }^{1}$ (i) \\ Laura Catherin García Rodriguez ${ }^{1}$ (i) \\ Ana Maria Amador Vargas ${ }^{1}$ \\ Sarita Nicole Pepinosa Lopez ${ }^{1}$ (i) \\ Ana Julia Carrillo Algarra ${ }^{1}$ (c) \\ Sandra Milena Hernández-Zambrano ${ }^{1}$ (i)
}

\begin{abstract}
Objective: To understand the experience of nurses and decision-makers when implementing the case management model in the care of patients with multimorbidity and their family caregivers. Method: Qualitative descriptive study including nurses and decision-makers and conducted in Bogotá, Colombia. Content analysis of field journals and semi-structured interviews carried out in 2019-2020 was performed. ATLAS-TI was used for data processing and analysis. Results: Six nurses and two decision-makers participated in the study. The following categories emerged: Nursing perception of care provided to patents with multimorbidity and their social environment; Nursing care experience in case management: role, contributions, and difficulties; and Implementation of the case management model in the Colombian context: implementation, impact, and role of providers. Conclusions: The case manager requires experience and skills to communicate properly. Case management improves quality of life, therapeutic adherence, use of health services and reduces overload.
\end{abstract}

DESCRIPTORS: Case management; Multimorbidity; Nursing; Caregivers; Qualitative research.

CÓMO REFERIRSE A ESTE ARTÍCULO:

Montañez RAC, Rodriguez LCG, Vargas AMA, Lopez SNP, Algarra AJC, Hernández-Zambrano SM. Análisis del modelo de gestión de casos para personas en situación de pluripatología y cuidadores familiares. Cogit. Enferm. [Internet]. 2021 [acesso em "colocar data de acesso, dia, mês abreviado e ano"]; 26. Disponível em: http://dx.doi. org/10.5380/ce.v26i0.79823. 
La transición epidemiológica y demográfica mundial, explica el aumento de Enfermedades Crónicas no Transmisibles (ECNT), asociadas a pérdida de años de vida productivos y alta carga familiar, social y económica que matan 41 millones de personas cada año mundialmente $(71 \% \text { de la mortalidad general })^{(1)}$. En Colombia más de 110 mil personas, fallecen por ECNT(2), quienes las sufren reportan que disminuye calidad de vida y adherencia terapéutica, genera deterioro funcional, interrumpe actividades familiares; aumenta reingresos hospitalarios, complicaciones y fragilidad ${ }^{(3)}$.

La multimorbilidad o pluripatología, requiere modelos de atención como Kaiser Permanente que estratifica las personas según riesgo, combina prevención, autogestión, gestión de la enfermedad y gestión de casos (GC) en pacientes de alta complejidad ${ }^{(4)}$. La GC es un rol de Práctica Avanzada en Enfermería, orientado a proveer servicios de salud a pacientes crónicos complejos y cuidadores principales para satisfacer sus necesidades, minimizar fragmentación de la atención, coordinar diferentes proveedores y mejorar continuidad asistencial(5-6).

A pesar de la efectividad del modelo de GC en resultados en salud, como controlar la situación clínica, disminuir sobrecarga del cuidador y polifarmacia, mejorar utilización de servicios de salud, coordinación asistencial, calidad de vida, y atención domiciliaria ${ }^{(7)}$, no se localizan reportes de su implementación y evaluación en el Sistema de Salud Colombiano.

El análisis cualitativo para evaluar el modelo de GC con población crónica compleja en Colombia determinó su orientación a: continuidad asistencial con coordinación entre niveles, profesionales y sectores; movilización de servicios socio-sanitarios; optimización de servicios; garantía de calidad en las transiciones y plan terapéutico individualizado; promueve empoderamiento y autonomía en la gestión asistencial de paciente y cuidador principal(8).

Dada la importancia de recoger evidencias cualitativas, el objetivo del estudio, es comprender la experiencia de enfermeras y profesionales de Entidades Administradoras de Planes de Beneficios (EAPB), frente a la implementación del modelo de GC para atender pacientes pluripatológicos y sus cuidadores familiares.

\section{MÉTODO}

Estudio descriptivo cualitativo, enfoque importante en investigación evaluativa, porque proporciona evidencias sobre mecanismos básicos para las intervenciones, examina el proceso de implementación de programas de atención en salud y mide resultados.

Participaron seis enfermeras entrenadas en GC y dos profesionales tomadores de decisión de dos EAPB. Criterios de inclusión: Enfermeras gestoras de caso, enfermera registrada para Colombia, experiencia mínima hospitalaria o comunitaria de dos años, con especialización o maestría, entrenadas para abordar pacientes crónicos complejos y cuidadores familiares; para tomadores de decisiones: profesional de salud asignado por la EAPB para implementar el modelo de GC.

La GC se realizó en Bogotá mediante visita domiciliaria, dirigida a 317 pacientes pluripatológicos y sus cuidadores durante 12 meses con cuatro componentes: Identificación del caso; Valoración personalizada; Diseño, ejecución y evaluación del plan de atención utilizando taxonomías NANDA (North American Nursing Diagnosis Association), NIC (Nursing Interventions Classification), NOC (Nursing Outcomes Classification), coordinación 
de intervenciones con otros proveedores, gestión de barreras administrativas; y Cierre del caso.

Muestreo intencional por conveniencia basado en criterios de inclusión con participación voluntaria. Se recogieron seis diarios de campo(9), de julio a diciembre del 2019 y ocho entrevistas semiestructuradas entre abril y mayo del 2020 para conocer la relación terapéutica, implementación del plan de cuidados (PC) , aceptabilidad y factibilidad del modelo en Colombia; en tomadores de decisión se exploró el rol de las EAPB en la implementación del modelo en Colombia. Se realizaron y grabaron las entrevistas por videollamada utilizando Google Meet, con duración promedio de 45 minutos, las transcribió una persona entrenada, quien firmó compromiso de confidencialidad.

Procesamiento y análisis de datos: a través de ATLAS-Ti versión 8 , se organizaron las transcripciones de entrevistas y diarios de campo, se realizó análisis de contenido siguiendo las primeras etapas de Bryant y Charmaz $^{(10)}$, que propone codificación abierta línea por línea para crear códigos; a través de memos reflexivos, se examinan, comparan y categorizan datos para agruparlos por relaciones de significado en redes de códigos. Esto permitió comparar, resaltar puntos claves y conexiones (co-ocurrencias) e interpretar resultados para construir categorías finales, privilegiando las más soportadas por citas en vivo según el software.

Consideraciones éticas: investigación de riesgo mínimo, cumplió Resolución 8430 de 1993 del Ministerio de Salud de Colombia(11); aval del Comité de Ética en Investigación en Seres Humanos del Hospital de San José (acta 9 de mayo 24 de 2017).

Participaron seis enfermeras gestoras y dos profesionales de EAPB (Medicina y Enfermería); edad promedio 34.2 años, especialización en Enfermería nefrológica del adulto, epidemiología, gerencia de instituciones de salud y maestría en salud pública o Enfermería. Los cargos de los tomadores de decisiones fueron: coordinadora de rutas de atención y profesional del modelo de salud.

Emergieron tres categorías, de las cuales surgieron subcategorías así:

\section{Categoría 1 - Percepción de Enfermería sobre el sujeto de cuidado pluripatológico y su entorno social}

Engloba la perspectiva de las enfermeras referente a definición del sujeto de cuidado, cuidador y entorno de cuidado. La subcategoría percepción del sujeto de cuidado y entorno describe la persona desde una perspectiva física, emocional y espiritual y como su entorno social influye en el desarrollo de la enfermedad; la subcategoría rol de cuidador y su sobrecarga, menciona el papel del cuidador para proveer cuidado en el hogar y la sobrecarga como factor de riesgo.

\section{Percepción sobre el sujeto de cuidado y su relación con el entorno}

La GC inicialmente es interpretada como invasión a su intimidad y entorno, algunos pacientes no querían acompañamiento ni seguían indicaciones por predisposición, prejuicios sobre el personal de salud, y escepticismo por experiencias previas desfavorables, esto dificulta la relación enfermera-paciente y la intervención, posteriormente la perspectiva del paciente cambia positivamente, mejora la comunicación y participa en su cuidado.

La pluripatología demanda tratamientos y hospitalizaciones que generan pérdida 
del trabajo; disminución de relaciones con amigos y familiares; aislamiento, deterioro del autoconcepto y autoconfianza. Algunas personas necesitaron atención psicológica, muchas evidencian necesidad de afecto. Espiritualmente, creer en un ser supremo genera, mayor afrontamiento, goce de experiencias de vida y comprensión o aceptación de la muerte.

Ella perdió su trabajo cuando le diagnosticaron la primera enfermedad [...] posteriormente se presentaron más enfermedades que afectaron su estado emocional, hasta llegar a un trastorno depresivo recurrente[...] ha perdido sus amigos... y reconoce que se ha aislado... tampoco tiene mucho tiempo, además de su rol como paciente pluripatológica es la cuidadora de sus padres y abuela. (Participante 2)

Las enfermeras refieren admiración al paciente pluripatológico quien, a pesar de su condición clínica y edad avanzada, se motiva a desarrollar actividades cotidianas y cuidarse para manejar su condición y potenciar su bienestar.

\section{Percepción de Enfermería sobre el rol del cuidador y su sobrecarga}

El cuidador es importante en la calidad de vida del paciente, brinda apoyo, acompañamiento, facilita el afrontamiento y manejo adecuado de la situación aportando al bienestar, independiente de condiciones socioeconómicas; algunos cuidadores son pacientes pluripatológicos, cuando esto ocurre en edades tempranas, desempeñan doble rol: paciente y cuidador.

[...] los cuidadores brindan atención en todas las actividades de rutina, desde la higiene diaria, alimentación, administración de medicamentos, hasta los difíciles trámites para obtener citas médicas [...]. (Participante 2)

Los familiares consideran que el Sistema de Salud Colombiano les delega el cuidado sin entrenamiento previo, esto ocasiona desconocimiento sobre el manejo de la enfermedad y dificulta satisfacer las necesidades del paciente, aunque exista interés y motivación para cuidar integralmente. El cuidador familiar asume actividades que suplen necesidades básicas, físicas, emocionales y sociales, u otras más complejas como la gestión administrativa de la atención por tanto existe riesgo de sobrecarga.

\section{Categoría 2 - Experiencia del cuidado de Enfermería en la GC: rol, aportes y dificultades}

Esta categoría analizó la experiencia enfermera respecto a su rol en el modelo. La subcategoría rol de enfermería enuncia competencias necesarias para cuidar durante la GC y la de aportes y dificultades, el beneficio de la experiencia a la vida personal y profesional de las gestoras y dificultades para implementar el modelo.

\section{Rol de Enfermería en la GC: percepción y competencias del profesional}

Las enfermeras reconocen la importancia de las taxonomías para implementar el PC individualizado, porque permite identificar necesidades con base en la valoración integral, plantear diagnósticos, e intervenciones para alcanzar los objetivos propuestos. La capacidad enfermera para cuidar integralmente define su rol en la GC, sus cuidados comprenden: acompañamiento domiciliario, educación a pacientes y cuidadores, seguimiento de intervenciones a través de llamadas telefónicas o videollamadas, proporcionar herramientas para empoderar pacientes y cuidadores y generar adherencia al tratamiento, reconocer el rol de cuidador y dar oportunidad de comunicar la sobrecarga, cuidados encaminados a fomentar bienestar físico, mental, emocional y articular los entornos de cuidado.

Durante la GC, se produjo el aislamiento preventivo debido al COVID-19, las gestoras con telefónicas o videollamadas orientaron las intervenciones para pacientes y cuidadores hacia medidas de protección personal como: distanciamiento, lavado de manos, uso de tapabocas e aislamiento. Dada la condición de los pacientes pluripatológicos, las enfermeras se enfrentaron a condiciones de fin de vida e intervinieron en el manejo de la 
pérdida, regulando la expresión de emociones durante el duelo. Además, reconocieron a las familias su importancia como cuidadores.

Por consenso el perfil de la gestora incluye experiencia clínica, comunitaria y administrativa, responsabilidad, liderazgo y habilidades comunicativas, para orientar, proporcionar cuidados integrales y empoderar a los pacientes en la toma de decisiones; recalcan que la GC sea dirigida por enfermería, dada su cercanía con los pacientes y su entorno, las habilidades para generar empatía y educar en salud. Se recomienda la articulación con otros sectores para mejorar la calidad de vida y el bienestar de la comunidad.

[...] experiencia clínica, docente, investigativa, con capacidad de liderazgo, comunicación y empatía [...] persona respetuosa con capacidad para transmitir información de manera clara [...]. (Participante 2)

Las gestoras resolvieron barreras relacionadas con entrega de medicamentos y oportunidad de citas médicas, además siguieron el avance del PC a pacientes y cuidadores en su domicilio o por llamada telefónica. Los prestadores de salud refieren que las enfermeras gestoras podrían participar en el reajuste del tratamiento farmacológico y direccionamiento a otras especialidades.

\section{Aportes a la vida personal, profesional y dificultades de la GC en la experiencia de cuidado}

Participar en GC para las enfermeras, significó autoconocimiento y aprendizaje; las vivencias con pacientes y familias les permitieron afianzar y obtener nuevos conocimientos para abordar vulnerabilidades y necesidades del paciente, y reflexionar sobre su autocuidado.

Además, incrementó su sensibilidad sobre el rol profesional en la evolución de la salud de una persona, la necesidad de actualizarse, mejorar sus intervenciones desde la evidencia para cumplir los objetivos del PC, la capacidad de escucha y aprendió sobre GC como modelo importante y necesario para la población colombiana, porque los inconvenientes en la atención dificultan el seguimiento del paciente por parte del equipo de salud y se traduce en deterioro, cuando se presentó durante la GC obstaculizó la atención oportuna y de calidad que generó reclamaciones por incumplimiento.

Las enfermeras reportan como dificultad la ubicación de participantes en lugares de difícil acceso y las actitudes agresivas que impiden las relaciones terapéuticas.

Al principio fue un poco complejo relacionarse con los pacientes, ubicarse, buscar la dirección [...]. (Participante 3)

Para un prestador la condición financiera y administrativa de su empresa ocasionó dificultades, por ello la población de esta EAPB fue menor, y reconoce como obstáculos la entrega de medicamentos y atención del usuario, fue imposible agilizar citas médicas. Un prestador considero que la no vinculación laboral de las enfermeras a la EAPB dificultó resolver barreras administrativas.

[...] no tener las gestoras dentro del aseguramiento, es una barrera, si estuviera en este, conocería la operación, y podría resolver con mayor facilidad [...]. (Participante 8)

\section{Categoría 3 - Implementación del modelo de GC en el contexto colombiano: implementación, efecto y rol de los prestadores}

Esta categoría incluye la perspectiva de los profesionales frente a la implementación del modelo de GC en Colombia. La primera subcategoría menciona como modalidades usadas visita domiciliaria y videollamadas, además de cambios en el sistema de salud para garantizar atención integral e integrada. La segunda enuncia beneficios percibidos por pacientes y cuidadores después de aplicar el modelo y la tercera describe el rol de los 


\section{Implementación de la GC en el contexto colombiano: modalidades y visión}

Durante la GC se realizó videollamada que según algunas enfermeras complementa la visita domiciliaria para seguimiento pero requiere recursos económicos para pagar internet, y saber usar esta tecnología, concuerdan que fue difícil que los adultos mayores aprendieran a utilizarla. Reportan como ventajas de la visita domiciliaria que: el paciente recibe atención en su hogar, permite mayor acercamiento, pacientes y cuidadores sienten más apoyo y acampamiento con la presencialidad, la enfermera conoce condiciones específicas del hogar para abordar necesidades reales y genera atención más personalizada con mejor adherencia al programa.

Debido a la contingencia del COVID-19, las gestoras continuaron la GC con llamadas telefónicas o video llamadas, refieren que comunicarse efectivamente no requiere presencialidad obligatoria; consideran que implementar el modelo de GC en Colombia aporta a la atención primaria en salud, permite abordaje efectivo de la población y facilita guiar pacientes y cuidadores por los servicios de salud evitando perder tiempo en trámites administrativos.

El sistema de salud colombiano presenta barreras para la GC, algunos pacientes asumen costos de su tratamiento, falla la articulación de la EAPB con la Institución Prestadora de Servicios de salud (IPS), existen trámites como autorización de medicamentos, que implican dirigirse a varios lugares a veces sin obtener respuesta, esto dificulta la continuidad del tratamiento y la intervención de enfermería. Implementar la GC, requiere medios, herramientas, profesionales y se debe analizar número de pacientes por gestora para que las intervenciones sean efectivas y logren el objetivo.

[...] va a ser un proceso de difícil adaptación, pero se podría desarrollar, porque es el interés hacerlo como enfermera, [...] se requieren medios y herramientas para hacer GC, puede ser demorado, pero se puede lograr. (Participante 4)

\section{Efecto del modelo de GC sobre pacientes y cuidadores}

Al indagar por el efecto percibido de la implementación de GC en Colombia, se identificaron beneficios para pacientes, cuidadores y enfermeras. En pacientes permitió mejorar aspectos de salud, respecto a adherencia al tratamiento, los pacientes se mantuvieron controlados, cumplieron la mayoría de recomendaciones, mejoraron la asistencia a controles médicos, disminuyó la asistencia al servicio de urgencias y aumentó la satisfacción de los pacientes con su sistema de salud.

Visualizar el rol del cuidador fue un logro, pues al hacerse explícito se fortaleció y demostró la importancia para el cuidado de los pacientes en el domicilio y como afecta directamente la calidad de vida y el proceso de enfermedad. Al identificar los cuidadores se integraron con el cuidado de sus familiares a través de intervenciones dirigidas a ellos, cuyo objetivo es proporcionarles apoyo en su adopción del rol y la identificación de sobrecarga, el modelo evidenció aspectos negativos que afectaban la relación entre ellos y su solución permitió mejores resultados en salud para paciente y cuidador.

[...] genera mayor adherencia al tratamiento, mayor conocimiento de la enfermedad disminuye costos, hospitalizaciones y asistencia a servicio de urgencia, [...] trabajar con la familia e integrarla fortalece las intervenciones para mejorar el proceso de enfermedad. (Participante 7)

\section{Prestadores de salud: rol y relación con la gestora}

En la implementación de la GC los prestadores de servicios de salud identificaron la población objeto del modelo y gestionaron las barreras administrativas, cada EAPB asignó un profesional encargado de realizar el enlace con las gestoras para conocer las necesidades y coordinar su resolución. Las gestoras participaron en las juntas de GC que 
realizaron las EAPB mensualmente para comunicar necesidades de los pacientes para que los prestadores pudieran solucionarlas oportunamente.

[...] yo era el enlace de la EAPB, para identificar los usuarios, la parte técnica, la articulación con las IPS, dentro de la EAPB con los diferentes grupos de riesgo y proveer información para poder gestionar las necesidades que encontraban las gestoras [...]. (Participante 7)

La relación prestadores-gestoras requiere buena comunicación para que la persona de enlace de la EAPB oriente las necesidades de los usuarios en diferentes áreas de la organización.

[...] una competencia importante es tener buena comunicación dentro de los diferentes procesos de la EAPB, conocer su ubicación, dónde gestionar esa dificultad que tiene el usuario y tener liderazgo, para apoyar y coordinar todo lo que necesita [...]. (Participante 7)

\section{DISCUSIÓN}

El desarrollo social y económico de Colombia favoreció un aumento de la esperanza de vida hasta 76 años según el Análisis de Situación de Salud 2019, uno de los más altos de América Latina y supera la media mundial de 72.5 años en $2018^{(12)}$. Los cambios sociodemográficos, culturales y de estilo de vida reflejan alta prevalencia de enfermedades crónicas, que generan problemas de salud con alto impacto social y financiero(13).

Las enfermeras consideran que el modelo es buena opción para garantizar la continuidad del paciente pluripatológico en el sistema de salud, entendida como el derecho a recibir atención de manera continua, sin interrupción por razones administrativas o económicas(14). La calidad de los servicios requiere integralidad, eficacia, eficiencia y continuidad para garantizar y proteger el derecho a la salud, de personas que demandan cuidados especializados institucionales y domiciliarios ${ }^{(15)}$.

La GC debe ser liderada por profesionales de Enfermería, definidos por el Consejo Internacional de Enfermeras ${ }^{(16)}$, como enfermera(o) titulada(o) con conocimientos expertos, habilidad para tomar decisiones, competencias clínicas o comunitarias para la práctica avanzada incluyendo habilidades de liderazgo y capacidad de articulación y mediación para orientar la resolución efectiva de las necesidades de pacientes pluripatológicos, así como con una formación especializada. Un estudio sugiere que la GC sea dirigida por enfermeras con conocimientos sobre modelos, métodos, técnicas de planificación, evaluación y gestión de calidad a través de liderazgo clínico y profesional y relaciones interprofesionales y consultoría, competencias propias de la práctica basada en evidencia(17).

La gestora debe brindar atención a las necesidades en hogares y comunidades, a través de integrar y coordinar los recursos del sistema de salud en los diferentes niveles de atención, por ello requiere competencias administrativas enfocadas a planificar, movilizar y gestionar recursos para mantener y mejorar la calidad de vida de la diada pacientecuidador desde una perspectiva holística(18). El efecto percibido por las Gestoras desvela resultados positivos en pacientes y cuidadores, porque mejoró la adherencia terapéutica y la sobrecarga del cuidador que se evidenció en disminución de ingresos a urgencias, admisiones, hospitalizaciones y pruebas diagnósticas. Los programas de GC conducen a mejoras en la capacidad funcional, mejor gestión de la medicación, reduce uso de servicios, reingresos hospitalarios, días de hospitalización y la institucionalización; esta estrategia aumenta las competencias para cuidar la díada paciente-cuidador.

Los cuidadores al integrarse directamente en las intervenciones enfermeras fortalecen su rol y pasan de experiencias frustrantes, agotadoras y estresantes a una buena en general ${ }^{(19)}$. Las enfermeras, describen fortalecimiento en su vida profesional y personal que 
varían según perspectiva, todas manifiestan que mejoran la calidad de vida de las personas y adquieren conocimientos y experiencias que las fortalecen como seres humanos.

La valoración del paciente pluripatológico evidencia necesidades físicas, espirituales, sociales, emocionales y psicológicas, la gestora diseña su PC basada en ellas, e incluye intervenciones de educación y asesoramiento para prevenir complicaciones y promover la salud facilitando la autonomía de pacientes y cuidadores, empoderándolos en su autocuidado. Se evidencia la importancia de la herramienta NANDA-NOC-NIC para integrar atención, porque apoya la toma de decisiones, facilita la selección de intervenciones que mejoran resultados en salud y permite efectuar seguimiento y evaluación de resultados enmarcado en el conocimiento técnico y científico ${ }^{(20)}$.

Respecto a las limitaciones del estudio, se declara que el análisis de la implementación del modelo de GC, se realizó con los sujetos que participaron en la intervención, factor que limitó número de participantes. Sin embargo, la triangulación de técnicas de recogida de datos (diarios de campo y entrevistas), permitió un análisis contextual del fenómeno de estudio, y la triangulación entre investigadores, quienes examinaron la información de forma independiente y posteriormente se reunieron para analizar en conjunto los aspectos más relevantes o los de no consenso, favoreció el rigor.

\section{CONCLUSIONES}

La enfermera gestora de casos requiere amplia experiencia clínica, comunitaria o administrativa; además de cualidades y aptitudes para comunicarse efectivamente con el paciente y cuidador para responder a las necesidades de la diada y mejorar su condición de salud. En cuanto al efecto percibido de la GC, se destaca mejora en la calidad de vida, en la adherencia terapéutica, asistencia a controles médicos y disminución de visitas a servicios de urgencias y hospitalizaciones. Los cuidadores al integrarse directamente con las intervenciones lograron fortalecer el rol de cuidador, y reducir la sobrecarga. Para las enfermeras la GC significó una nueva experiencia, crecimiento personal y profesional que les permitió desarrollar fortalezas frente al abordaje del sujeto de cuidado.

Este estudio aporta elementos claves sobre la implementación del modelo en Colombia, como el perfil del profesional, referenciación geográfica, contratación de la gestora por parte de la aseguradora, que permita la integración de todo el proceso de atención. De igual forma el modelo de GC para el país, es un reto en todos los escenarios, por ello se recomienda que las aseguradoras valoren su inclusión para alcanzar estándares de calidad de atención en salud en el ámbito domiciliario y clínico. El uso del lenguaje estandarizado en el cuidado de Enfermería permite al profesional tomar decisiones y elegir intervenciones eficaces para obtener mejores resultados.

\section{FINANCIACIÓN}

Este producto hace parte del proyecto "Efectividad de un modelo de gestión de casos para la provisión integral de servicios de salud a pacientes pluripatológicos" financiado por el Departamento Administrativo de Ciencia, Tecnología e Innovación de Colombia (Colciencias), a través de la convocatoria 777 para proyectos de Ciencia, Tecnología e Innovación en Salud - 2017, número de contrato 848-2017. 
1. Organización Mundial de la Salud. Enfermedades no transmisibles. [Internet]. 2018 [acceso 10 nov. 2020]. Disponible en: https://www.who.int/es/news-room/fact-sheets/detail/noncommunicable-diseases.

2. Organización Panamericana de la Salud. Las enfermedades no transmisibles (ENT), nuestro reto. [Internet]. 2018 [acceso 10 nov. 2020]. Disponible en: https://www.paho.org/col/index.php?option=com content\&view=article\&id=1756:las-enfermedades-no-transmisibles-ent-nuestro-reto\&ltemid=487.

3. Morales-Asencio JM, Martin-Santo SFJ, Kaknani S, Morilla-Herrera JC, Fernández-Gallego MC, García-Mayor S, et al. Living with chronicity and complexity: lessons for redesigning case management from patients' life stories - a qualitative study. J Eval Clin Pract. [Internet]. 2016 [acceso 10 nov. 2020]; 22(1):122-32. Disponible en: https://doi.org/10.1111/jep.12300.

4. Moyano AF, Lázaro JMM, Escalante MDM, Hernández MBA, Maroto IV. Modelos de atención al paciente pluripatológico. Rev. Clin Esp. [Internet]. 2017 [acceso 10 nov. 2020]; 217(6):351-8. Disponible en: https://doi.org/10.1016/j.rce.2017.03.003.

5. Zambrano SMH, Algarra AJC, Montañez RAC, Díaz OLC, Moreno SPC, Jiménez EG. Componentes del modelo de gestión de casos en la atención de la persona en condición de pluripatología y sus cuidadores familiares en Colombia. Paraninfo Digital. [Internet]. 2019 [acceso 12 nov. 2020]; 13(29):1-5. Disponible en: http://ciberindex.com/index.php/pd/article/view/e124.

6. Clíments GD, Álvarez CR, López AS, Gómez JAR, Aguirre JA, Gómez MBS. Evaluación del modelo de gestión de casos en Atención Primaria mediante enfermeras comunitarias de enlace. Ene. [Internet]. 2019 [acceso 15 feb. 2021]; 13(3):1336. Disponible en: http://scielo.isciii.es/scielo.php?script=sci arttext\&pid=S1988-348X2019000300006\&lng=es. Epub 24-Feb-2020.

7. Hernández-Zambrano SM, Mesa-Melgarejo L, Carrillo-Algarra AJ, Castiblanco-Montañez RA, ChaparroDiaz L, Carreño-Moreno SP, et al. Effectiveness of a case management model for the comprehensive provision of health services to multi-pathological people. J Adv Nurs. [Internet]. 2019 [acceso 12 nov. 2020]; 75(3):665-75. Disponible en: https://doi.org/10.1111/jan.13892.

8. Benjumea $C$ de la $C$. La contribución de la evidencia cualitativa al campo del cuidado y la salud comunitaria. Index Enferm. [Internet]. 2005 [acceso 12 nov. 2020]; 14(50):47-52. Disponible en: https:// scielo.isciii.es/scielo.php?script=sci_arttext\&pid=S1132-12962005000200010\&lng=es\&tlng=es.

9. Hernández-Zambrano SM, Montañez RAC, Algarra AJC, Rodriguez LCC. Análisis cualitativo del modelo de gestión de casos para la atención de personas en situación de pluripatología y sus cuidadores familiares. New Trends in Qualitative Research [Internet]. 2020 [acceso 15 feb. 2021]: 3:503-14. Disponible en: https://doi.org/10.36367/ntqr.3.2020.503-514.

10. Belgrave LL, Seide K. Coding for Grounded Theory. En: Bryant A, Charmaz K. The SAGE Handbook of Current Developments in Grounded Theory. SAGE publicatións. 2019; 167-85.

11. Ministerio de Salud (CO). Resolución 8430 de 1993. Por lo cual se establecen las normas científicas, técnicas y administrativas para la investigación en salud. Santafé de Bogotá D.C, 4 de octubre de 1993.

12. Ministerio de Salud y Protección Social (CO). Análisis de Situación de Salud 2019 [Internet]. Colombia: Imprenta Nacional de Colombia. 2020 [acceso 15 nov. 2020]. Disponible en: https://www.minsalud.gov. $\mathrm{co} / \mathrm{sites} / \mathrm{rid} /$ Lists/BibliotecaDigital/RIDE/VS/ED/PSP/asis-2019-colombia.pdf.

13. Banco Mundial. Esperanza de vida al nacer [Internet]. 2020 [acceso 15 nov. 2020]. Disponible en: https://datos.bancomundial.org/indicator/SP.DYN.LE00.IN.

14. Ley 1751 de 2015. Por medio de la cual se regula el derecho fundamental a salud y se dictan otras disposiciones. Santafé de Bogotá D.C, Congreso de Colombia, 16 febr 2015).

15. Yépez-Chamorro MC, Ricaurte-Cepeda M, Fajardo DMJ. Calidad percibida de la atención en salud 
en una red pública del municipio de Pasto, Colombia. Univ. Salud. [Internet]. 2018 [acceso 15 feb. 2021]; 20(2):97 -110. Disponible en: https://doi.org/10.22267/rus.182002.114.

16. Grover CA, Sughair J, Stoops S, Guillen F, Tellez L, Wilson TM, et al. Case Management Reduces Length of Stay, Charges, and Testing in Emergency Department Frequent Users. West J Emerg Med. [Internet]. 2018 [acceso 15 feb. 2021]; 19(2). Disponible en: https://dx.doi. org/10.5811\%2Fwestjem.2017.9.34710.

17. Miguélez-Chamorro A, Casado-Mora MI, Company-Sancho MC, Balboa-Blanco E, Font-Oliver MA, Román-Medina I. Enfermería de Práctica Avanzada y gestión de casos: elementos imprescindibles en el nuevo modelo de atención a la cronicidad compleja en España. Enferm Clin. [Internet]. 2019 [acceso 15 feb. 2021]; 29(2):99-106. Disponible en: https://doi.org/10.1016/j.enfcli.2018.08.005.

18. Zug KE, Cassiani SH de B, Pulcini J, Garcia AB, Aguirre-Boza F, Park J. Advanced practice nursing in Latin America and the Caribbean: regulation, education and practice. Rev Lat Am Enfermagem. [Internet]. 2016 [acceso 17 nov. 2020]; 24:e2807. Disponible en: http://dx.doi.org/10.1590/1518-8345.1615.2807.

19. Melo BRM, Hernández YV, Carrillo GM, Trujillo DKA. Efecto del programa Plan de egreso en la díada persona con enfermedad crónica y sus cuidadores familiares. Enferm Clin. [Internet]. 2017 [acceso 14 nov. 2020]; 28(1):36-43. Disponible en: https://doi.org/10.1016/j.enfcli.2017.09.006.

20. Adamy EK, Zocche DA de A, Almeida M de A. Contribution of the nursing process for the construction of the identity of nursing professionals. Rev Gaúcha Enferm.[Internet] 2020 [acceso 24 nov. 2020]; 41(esp):e20190143. Disponible en: http://dx.doi.org/10.1590/1983-1447.2020.20190143. 


\section{ANÁLISE DO MODELO DE GESTÃO DE CASOS PARA PESSOAS EM SITUAÇÃO DE PLURIPATOLOGIA E CUIDADORES FAMILIARES}

RESUMO:

Objetivo: compreender a experiência de Enfermeiros e tomadores de decisão na implementação do modelo de gerenciamento de caso no cuidado a pacientes com pluripatologias e seus cuidadores familiares. Método: estudo descritivo qualitativo com enfermeiras e profissionais tomadores de decisão, Bogotá - Colômbia. Análise de conteúdo de diários de campo e entrevistas semiestruturadas em 2019-2020. Processamento e análise de dados com ATLASTi. Resultados: participaram seis enfermeiras e dois profissionais tomadores de decisão. Categorias emergentes: Percepção da enfermagem sobre a temática do cuidado patológico múltiplo e seu meio social; Experiência assistencial de enfermagem no gerenciamento de casos: papéis, contribuições e dificuldades; Implementação do modelo de gestão de casos no contexto colombiano: implementação, efetivação e papel dos profissionais. Conclusões: Os gestores de casos requer experiência e habilidades para se comunicar adequadamente. O gerenciamento de casos melhora a qualidade de vida, a adesão terapêutica, a utilização de serviços de saúde e reduz a sobrecarga.

DESCRITORES: Gestão de caso; Multimorbidade; Enfermagem; Cuidadores; Pesquisa qualitativa.

\section{ANÁLISIS DEL MODELO DE GESTIÓN DE CASOS PARA PERSONAS EN SITUACIÓN DE PLURIPATOLOGÍA Y CUIDADORES FAMILIARES}

\section{RESUMEN:}

Objetivo: comprender la experiencia de Enfermeras y tomadores de decisión frente a la implementación del modelo de gestión de casos en la atención de pacientes pluripatológicos y sus cuidadores familiares. Método: estudio descriptivo cualitativo con enfermeras y profesionales tomadores de decisión, Bogotá - Colombia. Análisis de contenido de diarios de campo y entrevistas semiestructuradas en 2019-2020. Procesamiento y análisis de datos con ATLAS-Ti. Resultados: participaron seis enfermeras y dos profesionales tomadores de decisión. Categorías emergentes: Percepción de enfermería sobre el sujeto de cuidado pluripatológico y su entorno social; Experiencia de cuidado enfermero en la gestión de casos: rol, aportes y dificultades; Implementación del modelo de gestión de casos en el contexto colombiano: implementación, efecto y rol de los prestadores. Conclusiones: La gestora de casos requiere experiencia y competencias para comunicarse adecuadamente. La gestión de casos mejora calidad de vida, adherencia terapéutica, utilización de servicios sanitarios y reduce la sobrecarga.

DESCRIPTORES: Manejo de caso; Multimorbilidad; Enfermería; Cuidadores; Investigación Cualitativa.

Recibido en: 04/03/2021

Aprobado en: 30/03/2021

Editor asociado: Luciana Alcântara Nogueira

Autor correspondiente:

Sandra Milena Hernández Zambrano

Fundación Universitaria de Ciencias de la Salud - Bogotá, Colombia

E-mail: smhernandez3@fucsalud.edu.co

Contribución de los autores:

Contribuciones sustanciales a la concepción o diseño del estudio; o la adquisición, análisis o interpretación de los datos del estudio - Montañez RAC, Rodriguez LCG, Vargas AMA, Lopez SNP; Elaboración y revisión crítica del contenido intelectual del estudio - Montañez RAC, Rodriguez LCG, Algarra AJC, Hernández-Zambrano SM; Aprobación de la versión final del estudio que se publicará - Montañez RAC, Rodriguez LCG, Vargas AMA, Lopez SNP, Algarra AJC, Hernández-Zambrano SM; Responsable de todos los aspectos del estudio, asegurando las cuestiones de precisión o integridad de cualquier parte del estudio - Montañez RAC, Rodriguez LCG, Algarra AJC, Hernández-Zambrano SM. Todos los autores aprobaron la versión final del texto.

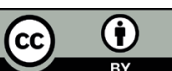

Copyright $\odot 2021$ Este es un artículo de acceso abierto distribuido bajo la licencia de atribución de Creative Commons, que permite el uso, distribución y reproducción sin restricciones en cualquier medio siempre que el artículo original esté debidamente citado. 